\title{
Improved Collectors for High Power Gyrotrons
}

\author{
Calabazas Creek Research, Inc. \\ 690 Port Drive \\ San Mateo, CA 94404 \\ (650) 312-9575 \\ RLI@CalCreek.com
}

Contract Number DE-FG02-06ER84441

June 25, 2006 through March 24, 2007 


\section{Introduction}

Electron cyclotron heating $(\mathrm{ECH})$ is well established as an effective mechanism for heating tokamak plasmas and is effective for controlling disruptive instabilities. Consequently, ECH heating systems are installed on tokamaks around the world and will be a critical component for the International Tokamak Experimental Reactor (ITER). ITER will require approximately $24 \mathrm{MW} \mathrm{CW}$ of RF power at $170 \mathrm{GHz}$. Currently, systems are being installed at Wendelstein 7X in Graifswald, Germany to provide approximately $16 \mathrm{MW}$ of RF power at $140 \mathrm{GHz}$, and the Doublet DIII-D reactor at General Atomics in San Diego will eventually use $10 \mathrm{MW}$ at $110 \mathrm{GHz}$.

Gyrotrons are in development and production around the world to provide this RF power. Currently, gyrotrons producing $1 \mathrm{MW}$ are available with $2 \mathrm{MW}$ sources in development.

These sources operate with pulses of several seconds or more to provide the heating power for the current generation of reactors. The typical electronic efficiency is approximately $33 \%$, which means that approximately $2 / 3$ of the electron beam power must be recovered and/or thermally dissipated in the collector. For a gyrotron producing $1 \mathrm{MW}$ of RF power, this means that approximately $670 \mathrm{~kW}$ of power remains in the electron beam after exiting the circuit.

A significant fraction of this power can be recovered by electrically depressing the collector voltage. Single-stage collectors are now routinely incorporated in MW-class gyrotrons to reduce the thermal loading and increase the operating efficiency. Typically, collector depression increases the total efficiency to approximately $50 \%$. The remaining power, approximately $500 \mathrm{~kW}$, is thermally dissipated.

Gyrotrons use very hollow electron beams for efficient interaction in the RF circuit. Typically, the electron beam in the circuit is extremely thin, as shown in Figure 1. As the beam exits the RF circuit and enters the collector, the beam expands as the magnetic field decreases, as shown in Figure $1^{1}$. Because the beam thickness remains relatively thin compared to the beam radius, the power is deposited over a small axial region in the collector. Consequently, the power density at the collector wall is quite high unless the collector radius is large or other techniques are employed to axially disperse the beam.

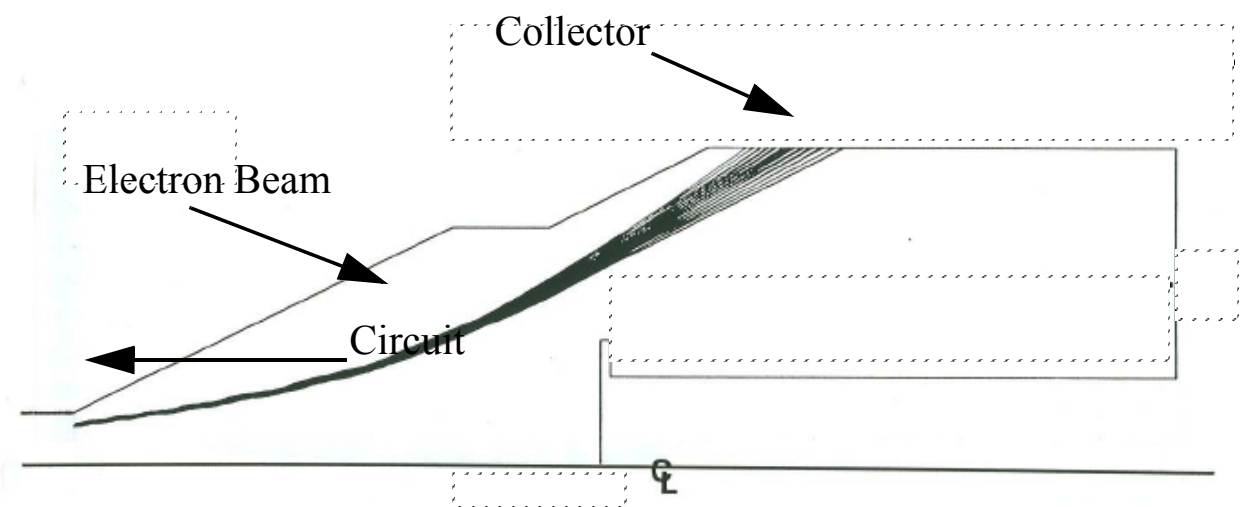

Figure 1. Electron beam simulation of a gyrotron collector 
The most obvious approach to reduce power density is to increase the radius of the collector. While this is effective, there are limitations due to cost and facilities. Collectors are basically large copper cylinders with water cooling to remove the thermal energy. These large cylinders are obtained as copper forgings available from a limited number of vendors, and the cost can be quite high. As the cylinders become larger, there is increasing concern for the quality of the copper with respect to voids and grain boundaries. Handling also becomes an issue with the increasing weight. Finally, gyrotrons must be baked to approximately $500^{\circ} \mathrm{C}$ prior to operation. The radial size of available ovens limits the maximum collector radius. Figure 2 shows a gyrotron produced by Communications and Power Industries, Inc. (CPI) for the DIII-D reactor at General Atomics. The collector portion of the gyrotron is indicated. It is the largest component of the gyrotron.

These high power gyrotrons use magnetic fields to axially spread the electron beam in the collector.

The current in one or more magnet coils is continuously cycled to axially move the electron beam along the surface. Figure 3 shows how the average power density is decreased by sweeping the elec-

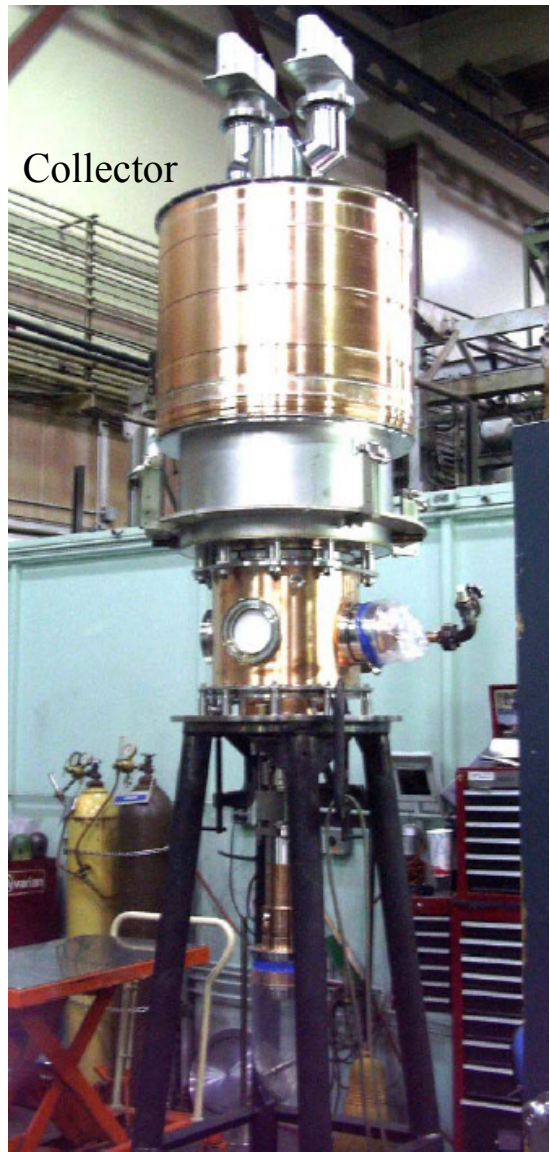

Figure 2. $110 \mathrm{GHz}, 1 \mathrm{MW}$ gyrotron produced by CPI tron beam ${ }^{2}$. The unlabeled curves indicate the deposited power density for single values of the sweep coil current, and the average line shows the resultant power density as a function of axial position. For this configuration, the average power is approximately $580 \mathrm{~W} /$ $\mathrm{cm}^{2}$. Power densities in excess of $600 \mathrm{~W} / \mathrm{cm}^{2}$ can lead to boiling in the water channels and thermal runaway, indicating that this configuration has very little margin against failure.

There are a number of issues with collector sweeping. Because the collector is manufactured from high conductivity copper, eddy currents prevent rapid sweeping of the magnetic field. Sufficient time must be allowed for the magnetic field to penetrate into the beam region. Any failure of the sweeping circuitry can lead to rapid destruction of the collector, so it is necessary to remove voltage to the gyrotron in this situation. This requires electronics to interlock the magnet power supply to the high voltage supply for the gyrotron.

Perhaps the most serious problem is that each section of the collector sees a pulse of heat with each sweep of the magnetic field. Pulse heating can lead to cyclic fatigue, and this mode of operation reduces the power density and stresses that the collector can withstand. 
Unfortunately, collector failures are a serious problem for high power

gyrotrons. Two CPI gyrotrons in operation at General Atomics and a 140 $\mathrm{GHz}$ gyrotron delivered to Graifswald in Germany both failed. Collector failure requires replacement of the most expensive component in the gyrotron, other than

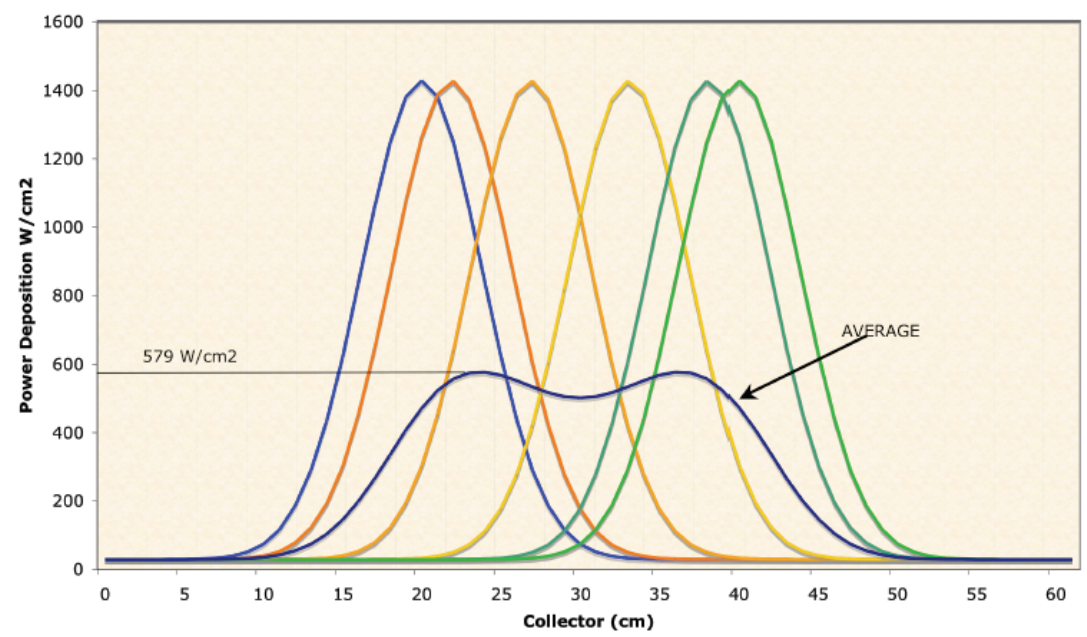

Figure 3. The effect of magnetic sweeping of the collector magnetic field to reduce power loading. Unlabeled curves are power densities at various values of the magnetic field.

the diamond output window. The rebuild and reprocessing cost can be $\$ 100 \mathrm{~K}$ or more, not to mention the costs incurred by the customer for removal, loss of use, and reinstallation.

The failure mechanisms are not well understood. Clearly, the primary cause is the high power densities deposited by the electron beam. Figure 4 displays a cross section of a collector that failed, showing a water cooling passage (center) and the vacuum surface (top). The arrow shows a crack in the material. There is also significant grain growth in the region. Grain growth is an indicator of material heating, though some grain growth is a normal result of gyrotron processing.

The failures appear to be located in regions of increased power density. The CPI gyrotrons incorporate resistance temperature devices (RTDs) to measure temperature at discrete locations of the collector. The failures appear to be located in regions displaying higher than average temperatures. Figure 5 shows the location of one of these failures. The plot indicates the azimuthal location along the

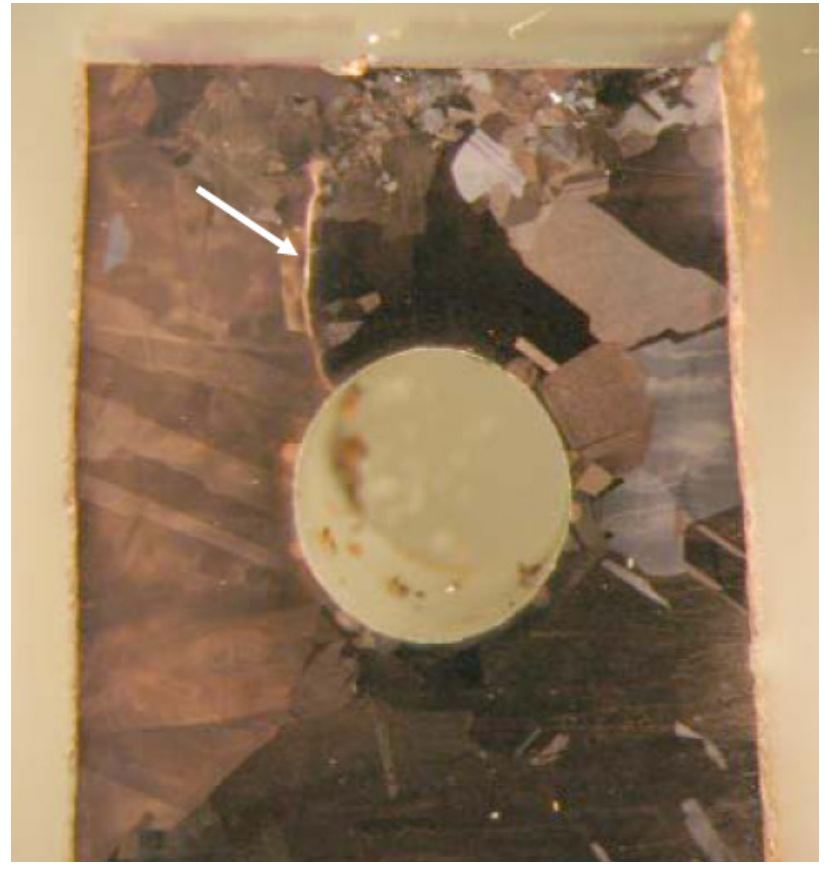

Figure 4. Cross section of gyrotron collector exhibiting a leak between a cooling channel and the vacuum region. The arrow indicates a crack through the copper. Significant grain growth is evident.

bottom axis and the axial location on the vertical axis. The temperature is indicated by the grey scale chart on the right, with white representing the highest temperature. The maximum power densities will occur nearer the bottom of the collector closest to the RF cir- 
cuit. Note also the azimuthal variation in temperature. This can be caused by variations in cathode emission or from azimuthal variations in the magnetic field and leads to additional uncertainty in the collector power density.

CPI performed extensive analysis of the failed collectors and provided the following conclusions and observations ${ }^{1}$ :

- The combination of pressure and temperature was sufficient to lead to permanent deformation of the collector.

- In most instances, cracking initiated at the cooling passages and propagated toward the inside diameter of the collector.

- The dominant failure mechanism that led to the collector failure was thermal fatigue - constraint was produced by the temperature gradient from the inside collector surface to the cooling passages.

- Metal loss due to vaporization was present on the inside surface of the collector.

LION COLLECTOR MAP AT GA--650 kW OUTPUT $78 \mathrm{kV}, 40 \mathrm{~A}$, ICOLL-DC $=16.9$ A Command, ICOLL-AC $=19.0$ A Command, $4 \mathrm{~Hz}, 16: 45--11 / 12 / 04$

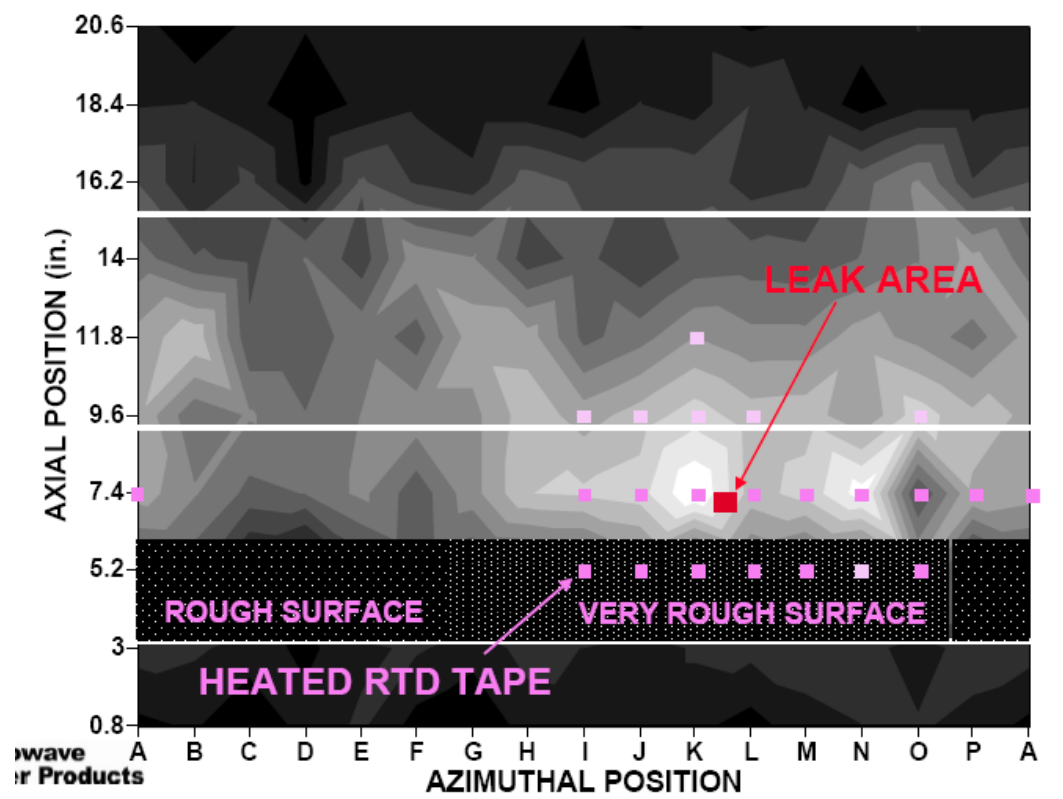

Figure 5. Power density plot for collector that failed

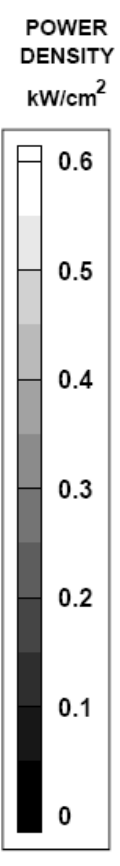

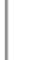
.

Phase I program reviewed the analysis of the failed collectors and obtained additional information of the failure mechanisms. This information was used to investigate alternative designs, materials, and processes to eliminate the failure modes. The initial goal was to reduce the maximum heat flux on any collector surface to less than $400 \mathrm{~W} / \mathrm{cm}^{2}$. As will be shown, simulations of designs examined during Phase I reduced the power densities to less than $500 \mathrm{~W} / \mathrm{cm}^{2}$. It appears that computer optimized simulations, proposed for Phase II, can achieve the $400 \mathrm{~W} / \mathrm{cm}^{2}$ goal.

In a previous SBIR program, Calabazas Creek Research, Inc. (CCR) developed a twostage depressed collector for MW-class gyrotrons ${ }^{3}$. A combination of adiabatic and nona- 
diabatic magnetic fields and collector voltages provided energy separation for the spent electron beam. The fields directed electrons with specific energies to appropriate surfaces to maximize the efficiency. That same technique was applied to single-stage or grounded collectors to direct high energy electrons to regions where the heat flux can be reduced by electrical or geometrical techniques. This technique is not currently used in gyrotron collectors.

The common use of collector depression provides an additional mechanism for reducing heat flux. In general, the primary focus of collector depression is to increase the operating efficiency, but in so doing, it also decreases the energy of the electrons. The amount of depression depends on the incoming energy of the slowest electrons and the subsequent generation of backscattered primary electrons. In most cases (but not all), it is the backscattered electrons that limit the depression that can be achieved. By developing techniques to trap backscattered electrons, increased depression can be achieved with subsequent reduction in thermal energy in the collector. All designs simulated during Phase I included consideration of secondary electrons. Trajectory codes can simulate up to three generations of secondary electrons.

The program explored alternative collector sweeping schemes. Axial collector sweeping was described above, so its advantages and disadvantages are well known. An alternative is azimuthal or transverse collector sweeping. In this approach, a series of coils are located between the collector and circuit with their axes radially outward from the gyrotron axis.

The currents in the coils are sequentially modified so that the electron beam is moved azimuthally about the gyrotron axis. There appear to be several advantages to this approach; however, discussions with Manfred Thumm of FZK in Germany indicate this approach did not solve the problem ${ }^{6}$. The Germans are currently examining other alternatives to azimuthal sweeping.

The program considered alternative materials to address the cyclic fatigue issue. While copper is extremely conductive, other materials are capable of withstanding higher heat loads. This program investigated plasma sprayed tungsten and tungsten copper with encouraging results. This will be discussed later in this proposal. Following discussions with Kevin Felch of Communications \& Power Industries, Inc. (CPI), it was determined that any new materials should be tested at high power densities prior to implementation into an actual gyrotron. Consequently, the Phase I program designed a test facility to allow high power, cyclic testing of potential collector materials. This facility is proposed for the Phase II program.

The program also investigated a diagnostic technique to evaluate the performance of collectors and provide more rapid and reliable interlock monitoring. Jeff Neilson of CCR conceived a real-time technique for monitoring collector temperatures using reflectometry. RF signals are launched through a dielectric rod inserted in the wall of the collector parallel to the heated surface. Using the temperature dependent dielectric properties of the rod, computational routines determine the temperature as a function of position. Unlike existing resistance temperature device (RTD) measurements, this technique could be used during full power operation and could be interfaced to interlock circuitry. The technique could detect anomalous collector operation, such as blockage of a cooling channel, and alert the user before significant damage could occur. During the Phase I program a test fixture was built and tested to determine feasibility of this concept. 
The program considered the fabrication and assembly cost in the Phase I studies. CPI was the principle industrial partner and contributed its years of experience and expertise and assisted with evaluating the effectiveness and cost. The efforts in this program were also coordinated with on-going efforts at CPI to ensure the research was complementary. CCR also discussed the Phase I research with Manfred Thumm of FZK, who asked to be kept informed of progress.

\section{Anticipated Public Benefits}

Successful completion of this program will eliminate a major failure mechanism in high power gyrotrons. This, in turn, will reduce cost by eliminating risk for the manufacturer. It will also reduce downtime and costs incurred by users when the gyrotron is removed from service for repair. An additional goal of the research will be to reduce the manufacturing cost of high power gyrotrons and the associated power supply and support systems. Successful completion could also result in more efficient gyrotrons.

\section{Degree to Which Phase I Demonstrated Technical Feasibility}

The technical tasks proposed for the Phase I program were as follows:

1. Examine existing analyses of gyrotron collector failures and determine the failure modes.

2. Investigate use of nonadiabatic magnetic fields to increase energy dispersion of the electron beam.

3. Examine alternative geometries to reduce transmission of backscattered electrons to critical surfaces to allow increased collector depression.

4. Investigate alternative materials.

5. Develop a real-time diagnostic for collector monitoring during operation.

6. Design a facility for testing collectors in the Phase II program.

\subsection{Examine existing analyses of gyrotron collector failures and determine the failure modes}

CCR personnel discussed the collector situation with Dr. Kevin Felch of CPI and Philipp Borchard during the first weeks of the program. Dr. Felch described the situation as currently understood and suggested actions to address the problem. Cyclic fatigue caused by the axial sweeping appears to be the primary cause of failure. This situation was essentially as described above and in the Phase I proposal. Philipp Borchard confirmed this information. Mr. Borchard performed the thermal and mechanical analysis for CPI. Based on these discussions, slight changes were made to Task 4, and the fundamental approach planned for Task 6 was modified. These tasks are described in detail below.

\subsection{Investigate use of nonadiabatic magnetic fields to increase energy dispersion of the electron beam}

During CCR's multi-stage depressed collector program, nonadiabatic fields were effective in sorting electrons by energy and dispersing them within the collector. Consequently, this 
technique was applied to a single-stage collector similar to those used on the General Atomic gyrotrons. One approach was to take the two-stage design developed earlier and simulate it as a single-stage collector. Essentially, both stages were energized to the same voltage. This simulation is shown in Figure 6, and the associated heat profile is shown in Figure 7.

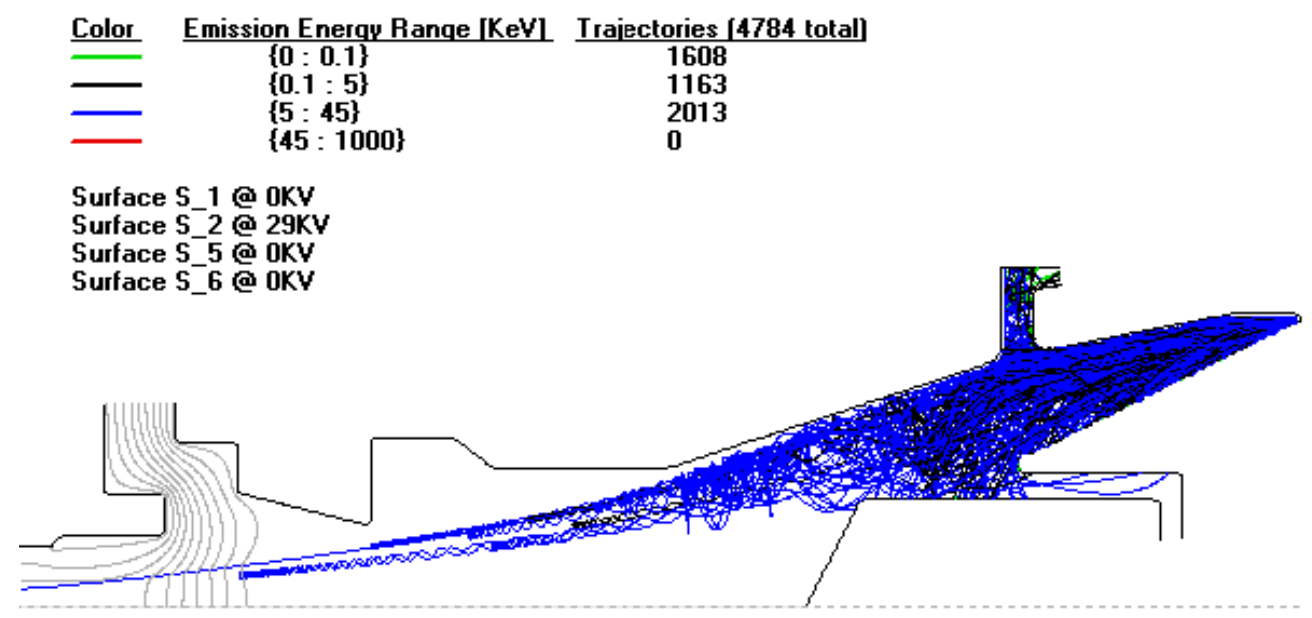

Figure 6. Two-stage collector simulation with both stages at $29 \mathrm{kV}$ with three generations of secondary electrons.

As Figure 7 indicates, this design reduces the peak heat dissipation to less than $500 \mathrm{~W} / \mathrm{cm}^{2}$. Note the strip width for collecting the power densities is 0.5 inches. Any increase in this value would reduce the peak power density even further. This value is significantly lower than the average value in the swept collectors $\left(580 \mathrm{~W} / \mathrm{cm}^{2}\right)$.

While the two-stage collector design should achieve the basic goal of the program, it is mechanically complex to allow for the additional ceramics. Con-

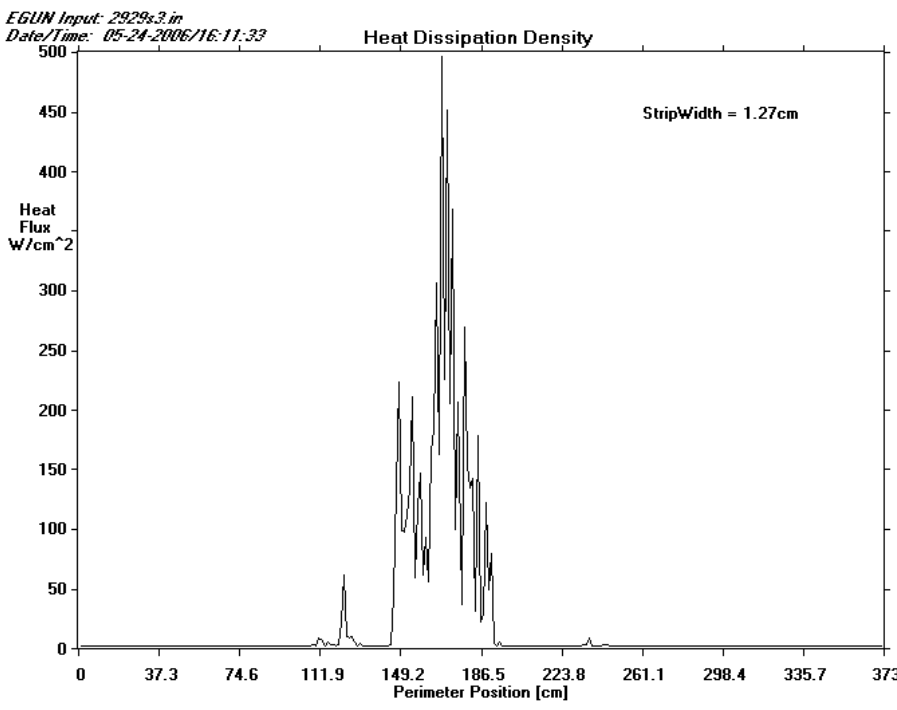

Figure 7. Heat dissipation profile for two-stage collector with both stages at $29 \mathrm{kV}$ sequently, the program explored simpler designs to achieve the reduced power densities.

Figure 8 shows trajectories using a simplified version of the collector in Figure 6. This design was also modified to duplicate the transition between body and collector voltage in 
the 1.5 MW gyrotron operating at General Atomics. The spent beam data is also from the 1.5 MW design.

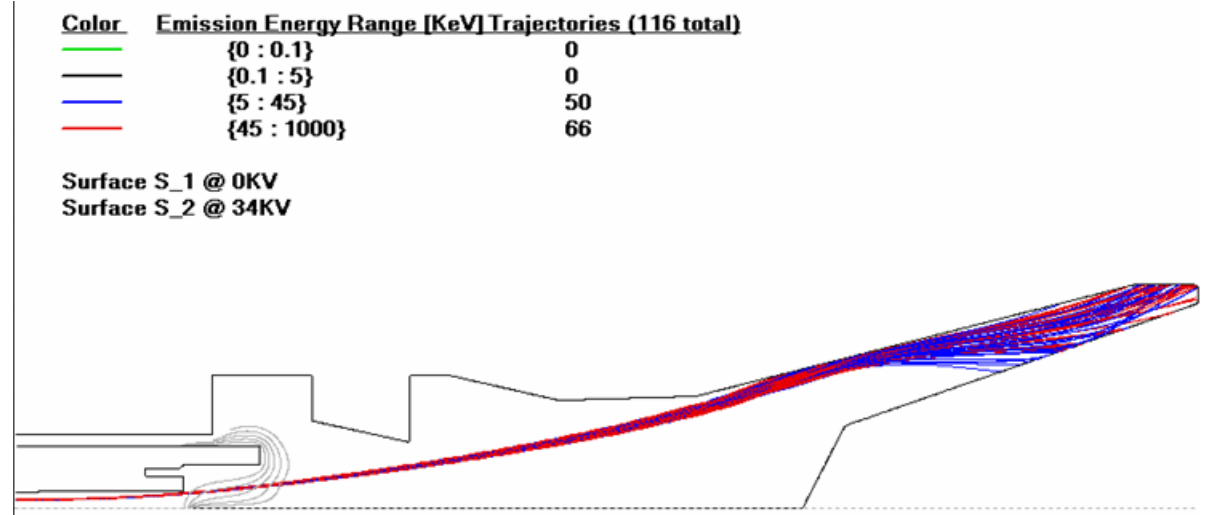

Figure 8 . Collector simulation with $34 \mathrm{kV}$ of voltage depression, including primary electrons (red) and first generation of secondary electrons (blue)

The predicted power densities depend on several factors. To account for the limited number of trajectories that can be simulated, one averages the power densities over regions of the collector. Basically, one divides the surface into 'bins' and quantifies the power collected in the individual bins. Otherwise, peaks would be extremely high where a trajectory strikes the surface and zero where a trajectory does not strike the surface. This is obviously not indicative of reality where an essentially unlimited number of electrons strike the surface. The larger the bin size, the greater the smoothing of the power densities. The bin width used is chosen based on the number of trajectories and some consideration for axial heat spreading that occurs naturally in the collector. Typically, this value is between $1.27 \mathrm{~cm}(0.5 \mathrm{inch})$ and $2.54 \mathrm{~cm}(1 \mathrm{inch})$. Figure 9 shows the dissipation profiles for the geometry of Figure 8 with two different bin widths. Note that the peak power density goes
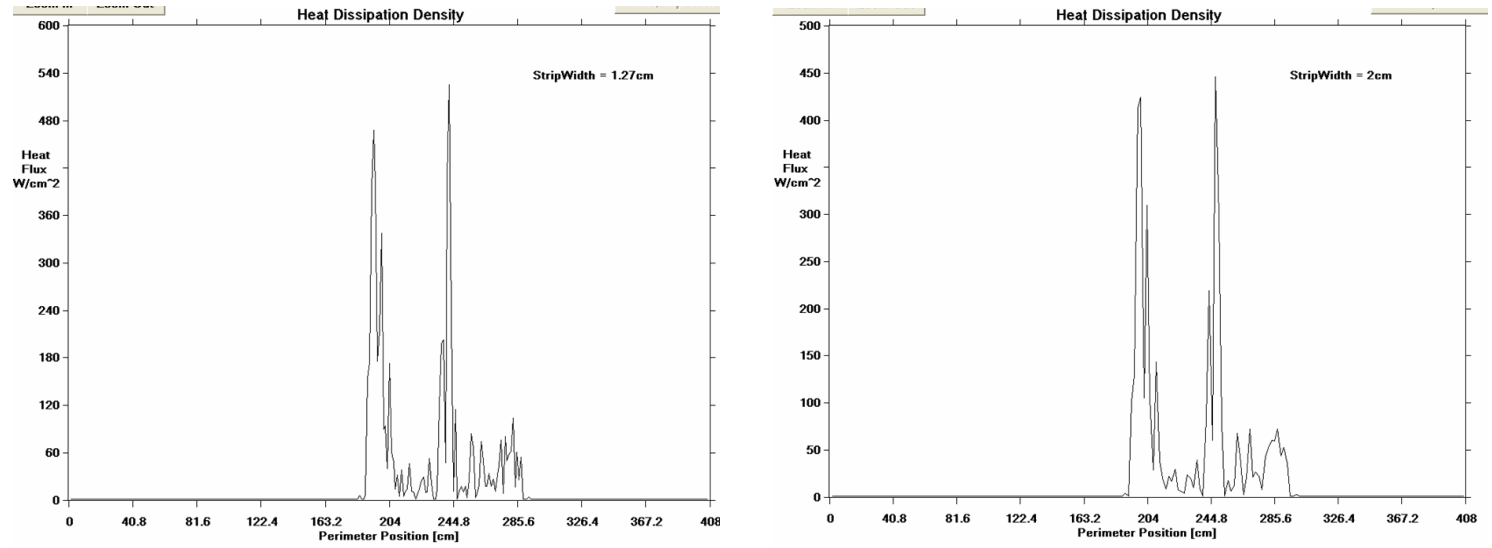

Figure 9. Heat dissipation profiles for geometry of Figure 8 with $28 \mathrm{kV}$ of voltage depression. Bin width for left profile is $1.27 \mathrm{~cm}$ and for right profile is $2.0 \mathrm{~cm}$

from approximately $540 \mathrm{~W} / \mathrm{cm}^{2}$ to approximately $450 \mathrm{~W} / \mathrm{cm}^{2}$ when the bin width is increased from $1.27 \mathrm{~cm}$ to $2.0 \mathrm{~cm}$. 
The power density also depends on the voltage depression. In the Phase I simulations, depressions approaching $37 \mathrm{kV}$ were achieved before there was significant reflection of electrons back toward the circuit. In actual operation, however, depression voltages on the order of $27 \mathrm{kV}$ were the maximum that could be achieved before significant current was measured in the circuit. Figure 10 shows the dissipation when the depression voltage is increased to $34 \mathrm{kV}$ for a bin width of $1.27 \mathrm{~cm}$. In this case, the peak power density is reduced to approximately $380 \mathrm{~W} / \mathrm{cm}^{2}$.

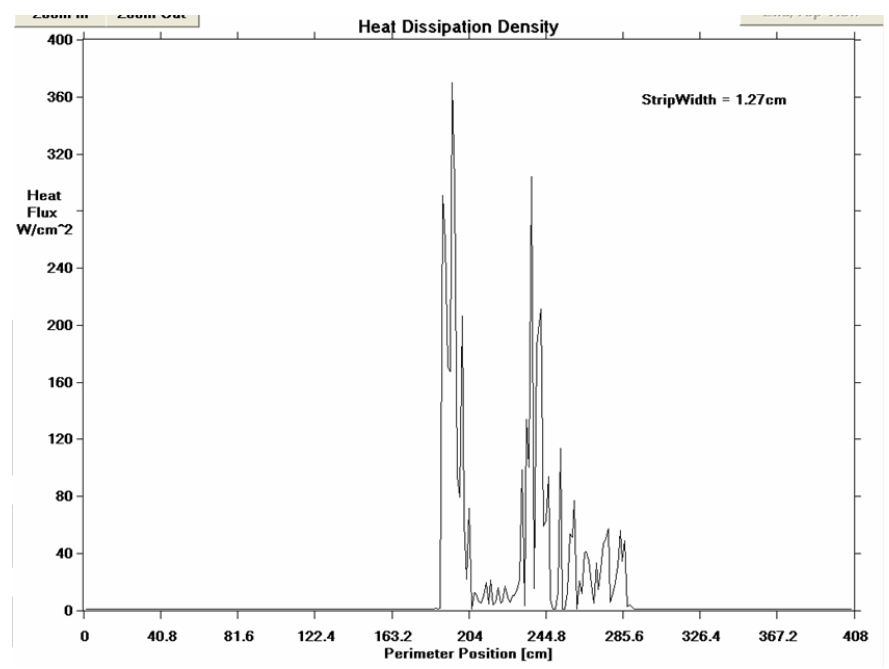

Figure 10. Heat dissipation profile for geometry of Figure 8 with $34 \mathrm{kV}$ of voltage depression and $1.27 \mathrm{~cm}$ bin width

Note that all these peak values are less than the average power density achieved with collector sweeping $\left(580 \mathrm{~W} / \mathrm{cm}^{2}\right)$. Also note that copper can tolerate significantly higher power densities under continuous operation than cyclic conditions. Therefore, the margin of safety is significantly higher for this geometry and mode of operation simulated than for axially swept operation.

To simulate 3D effects in the collector, CCR explored advanced simulations using its 3D finite element, adaptive mesh charged particle code, Beam Optics Analysis (BOA). The region from the quasi-optical launcher through the mirror region is not axially symmetric. The impact of these asymmetries on collector operation has not previously been simulated. CCR generated a solid model with a simple collector, as shown in Figure 11. Note

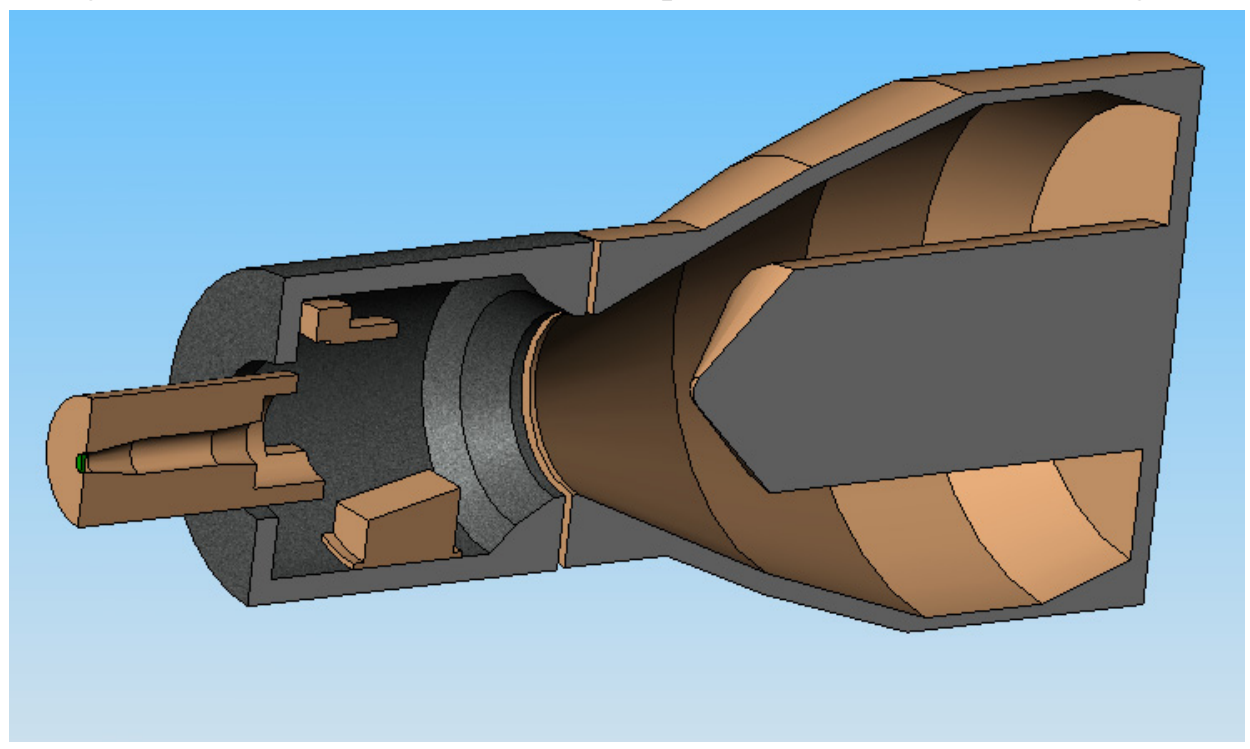

Figure 11. Sliced solid model of 8115 gyrotron interface and simple collector. Launcher and mirrors are included. 
that the model includes the location of the quasi-optical mirrors. The interface between body potential and collector potential occurs at the location indicated.

Figure 12 shows trajectories from a small, azimuthal region of the electron beam. This simulation allows one to visualize the rotation of the electron trajectories as they propagate through the field of the collector.

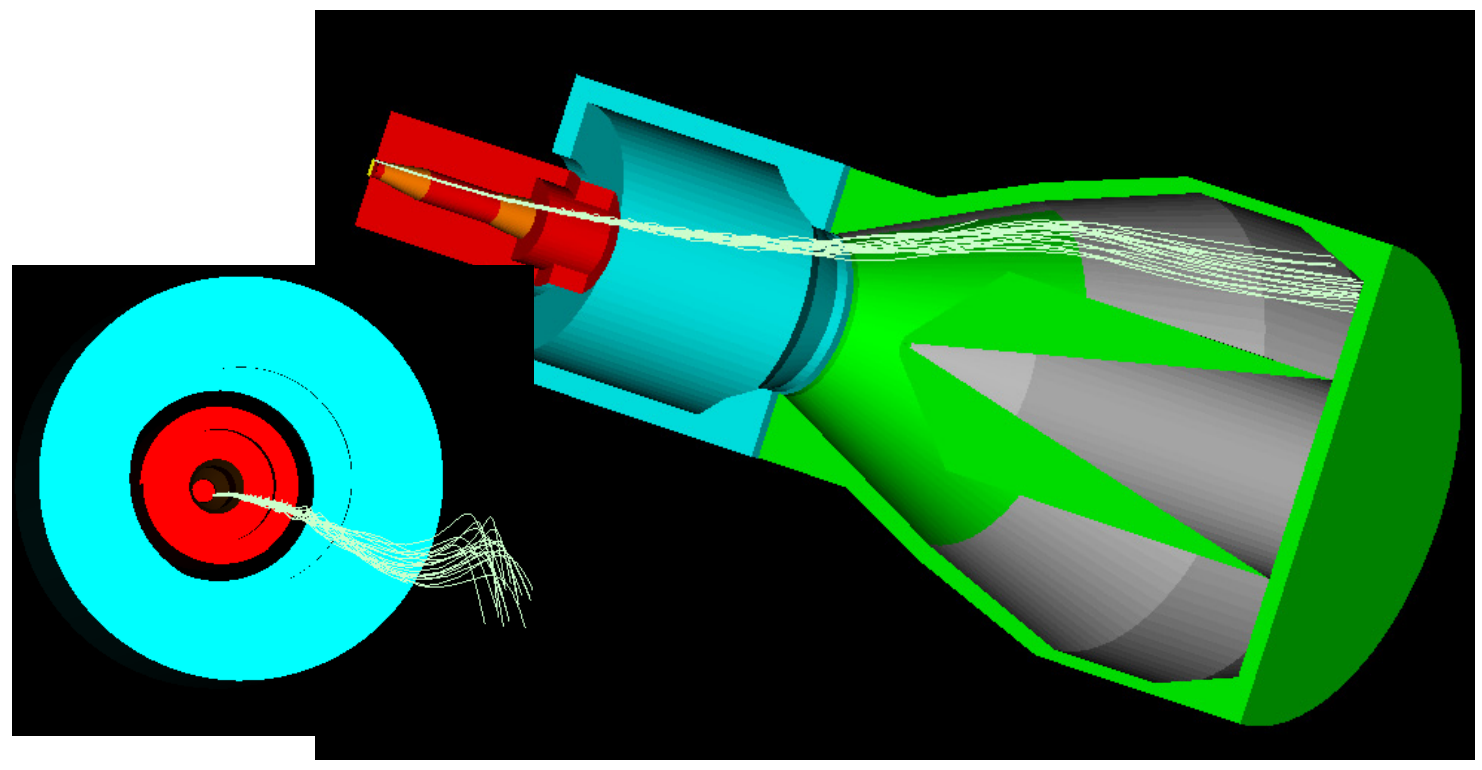

Figure 12.3D simulation of electrons at one azimuthal location into the gyrotron collector of Figure 11. Collector is hidden in end view in the left image.

Figure 13 shows a simulation with approximately 500 trajectories randomly distributed around beam azimuth. The figure also shows a simulation with three generations of secondary electrons. While these simulations provided some useful information, BOA does not currently allow calculation of power densities. This capability is currently being added and will be available for the Phase II program.
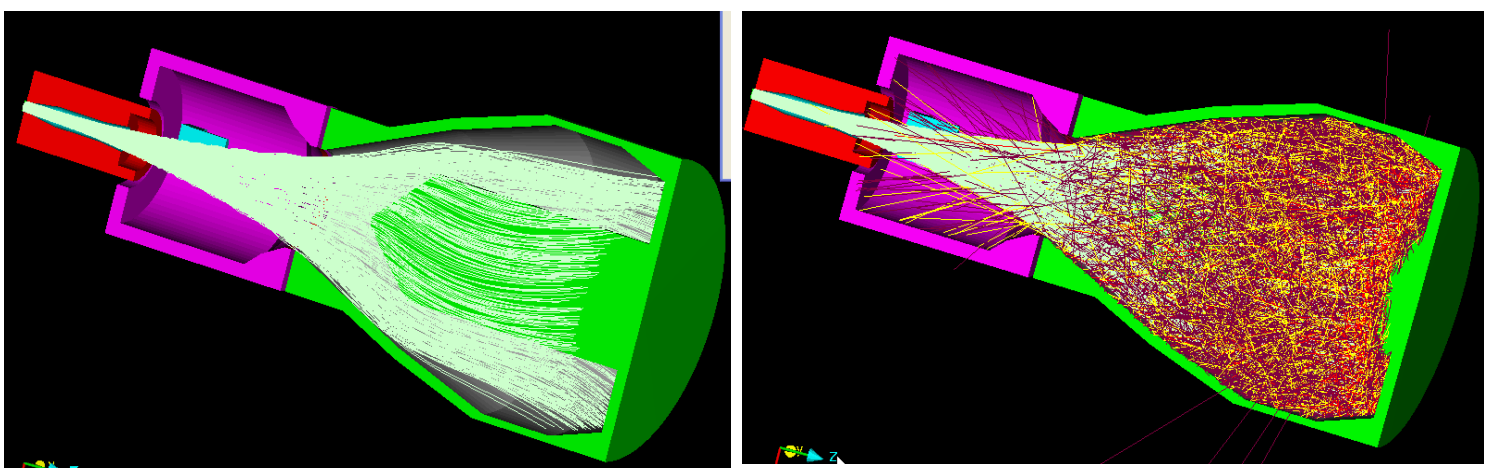

Figure 13. Simulation of configuration of Figure 11 with approximately 500 particles. Left image shows primary electrons, and right image includes three generations of secondary electrons. Secondary electrons shown leaving the collector are caused by a plotting error. 
While the goal of $400 \mathrm{~W} / \mathrm{cm}^{2}$ was not definitively achieved, the Phase I program clearly demonstrated that this is feasible. The simulations were performed with manual exploration of parameters. The Phase II program proposes to utilize computer optimized design to further reduce the power density. Computer optimization can explore significantly larger parameter space, so CCR is confident that the goal can be achieved.

\subsection{Examine alternative geometries to reduce transmission of backscattered electrons to critical surfaces to allow increased collector depression}

Because the performance of the collector depends on the impact of backscattered electrons, this task was incorporated into Task 2.2. Simulations indicated that the geometry shown in Figure 8 could incorporate $34 \mathrm{kV}$ of collector depression before backscattered electrons propagated to the body. Figure 14 shows the second and third generation of backscattered electrons for the geometry of Figure 8 . Note that only a single ray for the third generation reaches the left boundary of the problem. Third generation rays carry extremely low currents. This indicates the maximum depression voltage achievable before electrons begin propagating to the body.

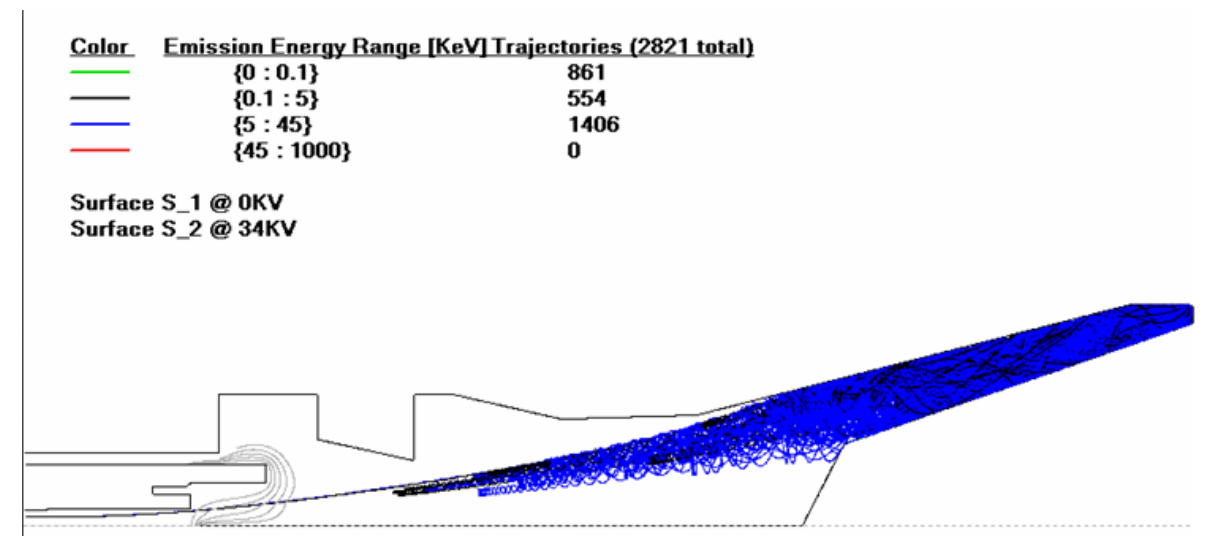

Figure 14. Second (blue) and third (black) generation of backscatter electrons with $34 \mathrm{kV}$ of collector depression

Note that most all backscattered electrons are collected within the coaxial section of the collector. Figure 9 indicates that all first generation backscattered electrons are collected in this region. Note the change in the collector geometry from that shown in Figure 6. The Phase I program determined that narrowing the region between the inner and outer collector surfaces significantly reduced secondary propagation toward the body.

\subsection{Investigate alternative materials}

A cause of the cyclic fatigue problem in the collector is the low strength of pure copper. While it provides superior thermal and electrical properties, its mechanical strength is not high. CPI is currently pursuing a form of copper containing alumina, called glidcop. This material has lower thermal conductivity but significantly higher mechanical strength than pure copper. Because the collectors are large diameter cylinders, the raw material must be forged to the approximate dimensions. This is not only time consuming, but also expensive. Glidcop does, however, appear to offer a potential solution to the problem. 
This program pursued an alternative approach where hardened materials are plasma sprayed to the copper surface. CCR uses plasma spraying to deposit titanium dioxide to the interior surface of its high power waterloads. Coatings up to approximately 0.125 inches can be applied, depending on the materials. For example, tungsten is plasma sprayed on jet engine turbine blades to significantly enhance the thermal mechanical performance. These loads can be significantly higher than those in gyrotron collectors.

To explore the potential of this approach, copper surfaces coated with tungsten were thermomechanically modeled. Of particular interest was the transient performance when thermally cycled. The ANSYS model is shown in Figure 15. For consistency with other cyclic fatigue simulations performed by CPI, the peak power density was cycled at a frequency of $8 \mathrm{~Hz}$ between 40 and $100 \%$ of the peak power density of $2.55 \mathrm{~kW} / \mathrm{cm}^{2}$. Figure 15 also shows the temperatures achieved for this heat load.
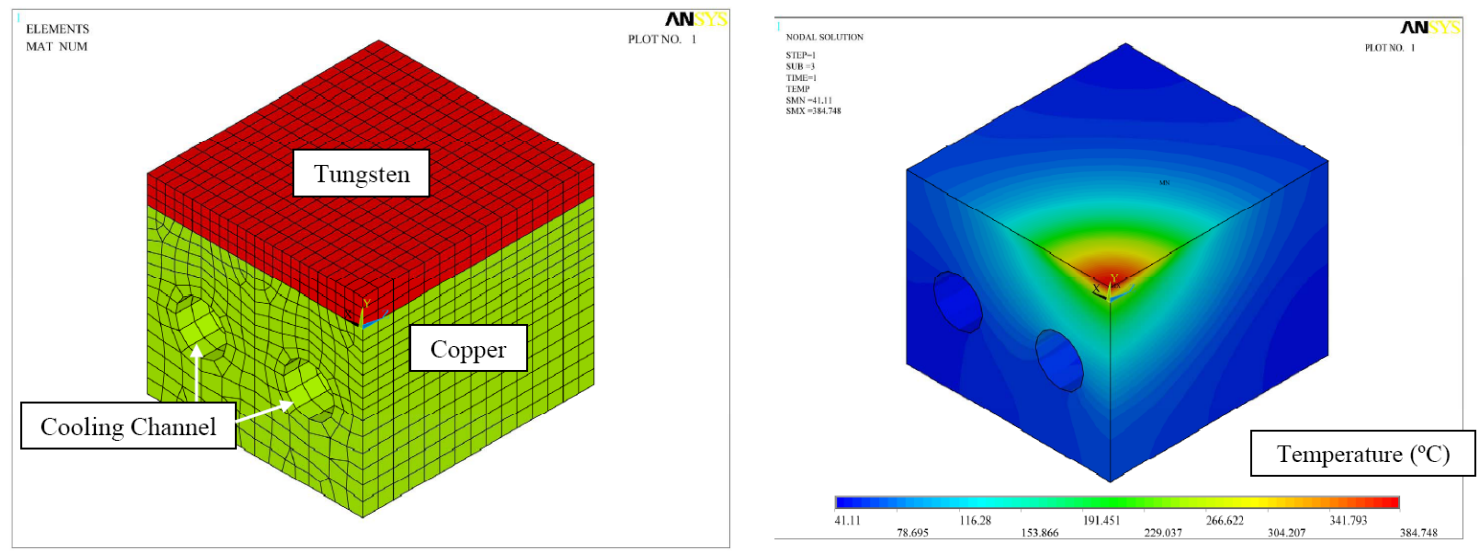

Figure 15. ANSYS model of a tungsten coated copper collector (left) and temperature contours when subjected to heat load (right) for 0.050 inch tungsten coating

The results are summarized in Figure 16. Note that Case 1 is for the current configuration with no tungsten coating. The simulations indicate that the number of cycles to failure increases by approximately one order of magnitude when there is a tungsten coating present.

\begin{tabular}{|l|c|c|c|c|}
\hline Case & 1 & 2 & 3 & 4 \\
\hline Coating Thickness (in) & 0 & 0.020 & 0.050 & 0.100 \\
\hline Coating Material & $\mathrm{W}$ & $\mathrm{W}$ & $\mathrm{W}$ & $\mathrm{W}$ \\
\hline Power Density $\left(\mathrm{kW} / \mathrm{cm}^{2}\right)$ & \multicolumn{5}{|c|}{2.55} \\
\hline Peak Temperature $\left({ }^{\circ} \mathrm{C}\right)$ & 304.9 & 371.9 & 451.7 & 538.9 \\
\hline Copper Strain Range $(\%)$ & 0.0542 & 0.0136 & 0.0140 & 0.0024 \\
\hline Tungsten Peak Stress (MPa) & - & 290 & 221 & 290 \\
\hline Tungsten Yield Stress (MPa) & \multicolumn{5}{|c|}{$>500$} \\
\hline Expected Cycles to Failure & $>1 \mathrm{e} 7$ & $>1 \mathrm{e} 8$ & $>1 \mathrm{e} 8$ & $>1 \mathrm{e} 8$ \\
\hline
\end{tabular}

Figure 16. Results of cyclic performance of various thickness of tungsten on copper 
The temperature of the collector surface as a function of time is shown in Figure 17. The results indicate that the surface temperature is higher for the tungsten coated surface, since the thermal conductivity is lower in the tungsten than in the copper.

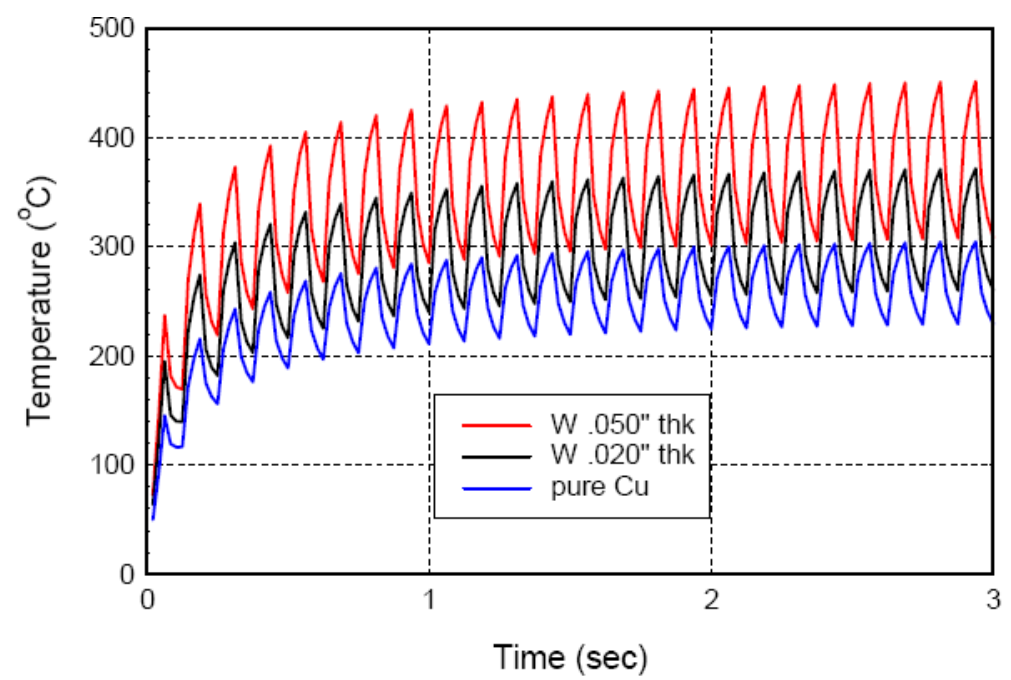

Figure 17. Transient thermal analysis of the collector section. Plot shows peak surface temperature of the surface versus time with sweep frequency of $8 \mathrm{~Hz}$.

Figure 18 shows why the cyclic performance is improved for the coated surface. This plot shows the temperature as a function of depth into the surface. While the surface temperature for the tungsten coated surface is significantly higher, the copper temperature is lower than for a pure copper collector. The increased strength of the tungsten allows it to tolerate the higher temperatures longer than the pure copper collector at a lower surface temperature.

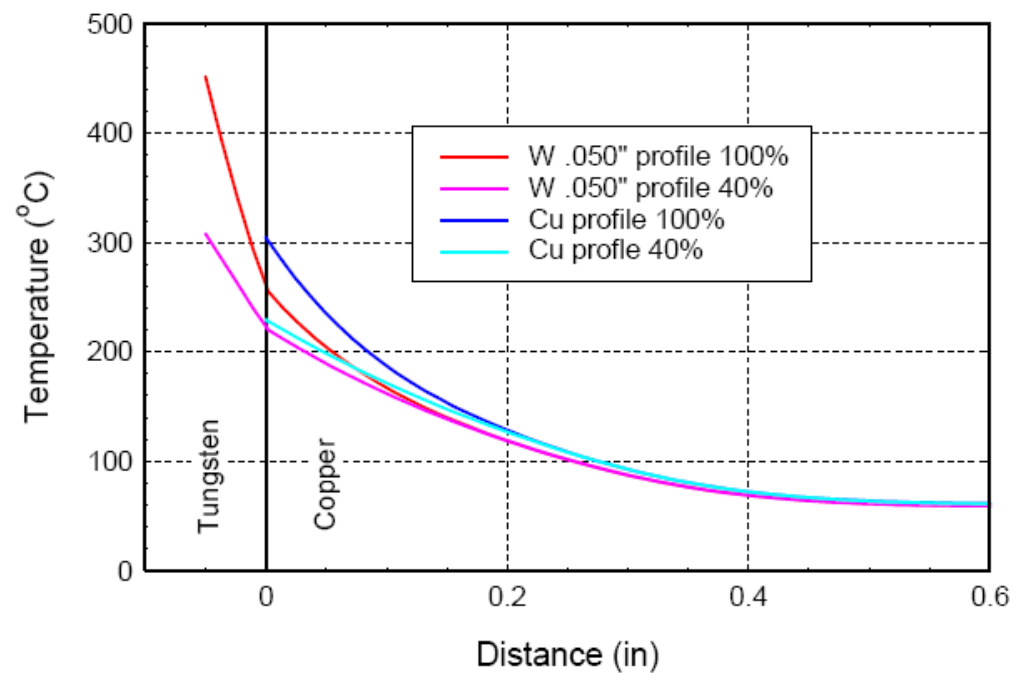

Figure 18. Temperature profiles at sweep extremes for pure copper surface and a section of 0.050 tungsten coating 
These results indicate that the lifetime can be increased for swept copper collectors with a tungsten coating. The performance is not improved, however, for on/off cycling of the collector. In fact, there is significant degradation in the expected lifetime. This occurs whenever the electron beam is turned off/on and the power density goes from zero to $100 \%$ of the nominal value. These results are presented in Figure 19. The decrease in lifetime is caused by the large difference in thermal expansion between the copper and tungsten.

\begin{tabular}{|l|c|c|c|c|c|}
\hline Case & 1 & 2 & 3 & 4 & 5 \\
\hline Coating Thickness (in) & 0 & 0.020 & 0.050 & 0.100 & 0.020 \\
\hline Coating Material & - & $\mathrm{W}$ & $\mathrm{W}$ & $\mathrm{W}$ & WCCo \\
\hline Peak Power Density $\left(\mathrm{kW} / \mathrm{cm}^{2}\right)$ & \multicolumn{5}{|c|}{1.78} \\
\hline Film Coefficient $\left(\mathrm{kW} / \mathrm{m}^{2} \mathrm{~K}\right)$ & \multicolumn{7}{|c|}{0.6} \\
\hline Copper Thickness $(\mathrm{in})$ & - & 320.2 & 384.7 & 468.5 & 362.7 \\
\hline Peak Coating Temp. $\left({ }^{\circ} \mathrm{C}\right)$ & 271.3 & 261.5 & 244.9 & 216.5 & 264.8 \\
\hline Peak Copper Temp. $\left({ }^{\circ} \mathrm{C}\right)$ & 124.8 & 122.2 & 118.0 & 110.4 & 123.2 \\
\hline Wet Wall Temp. $\left({ }^{\circ} \mathrm{C}\right)$ & - & 288 & 218 & 214 & 420 \\
\hline Coating von Mises Stress $(\mathrm{MPa})$ & 0.109 & 0.232 & 0.243 & 0.205 & 0.233 \\
\hline Copper Strain Range $(\%)$ & $1 \mathrm{e} 7$ & $2.7 \mathrm{e} 5$ & $2.2 \mathrm{e} 5$ & $4.7 \mathrm{e} 5$ & $2.6 \mathrm{e} 5$ \\
\hline Expected Cycles to Failure &
\end{tabular}

Figure 19. Results for on/off cycling of tungsten coated copper

To address this issue, CCR personnel worked with Plasma Coating Corp. to develop a process for plasma spraying a mixture of tungsten and copper. The expectation was that mixing the materials would provide the increased strength without stresses induced by larger thermal expansion differences. This could provide capability similar to glidcop but at a significantly reduced cost. Standard copper collectors could be easily sprayed with the tungsten copper mixture.

Simulation results for the tungsten copper coatings for on/off operation are provided in Figure 20 . The program simulated a mixture containing $75 \%$ tungsten and $24 \%$ copper and a mixture of equal parts tungsten and copper. The on/off operation results indicate that the 75-25 mixture resulted in an 80\% decrease in lifetime, while the 50-50 mixture provided a slightly increased lifetime (33\% increase).

\begin{tabular}{|l|c|c|c|c|}
\hline Case & 1 & 2 & 3 & 4 \\
\hline Coating Thickness (in) & 0 & 0.020 & 0.020 & 0.020 \\
\hline Coating Material & - & WCu 50/50 & WCu 75/25 & W \\
\hline Peak Power Density $\left(\mathrm{kW} / \mathrm{cm}^{2}\right)$ & \multicolumn{5}{|c|}{1.78} \\
\hline Film Coefficient $\left(\mathrm{kW} / \mathrm{m}^{2} \mathrm{~K}\right)$ & \multicolumn{5}{|c|}{0.6} \\
\hline Copper Thickness $(\mathrm{in})$ & - & 298.7 & 312.7 & 320.2 \\
\hline Peak Coating Temp. $\left({ }^{\circ} \mathrm{C}\right)$ & 271.3 & 258.3 & 260.6 & 261.5 \\
\hline Peak Copper Temp. $\left({ }^{\circ} \mathrm{C}\right)$ & 124.8 & 121.4 & 122.0 & 122.2 \\
\hline Wet Wall Temp. $\left({ }^{\circ} \mathrm{C}\right)$ & - & 203 & 218 & 288 \\
\hline Coating von Mises Stress (MPa) & 0.109 & 0.105 & 0.146 & 0.232 \\
\hline Copper Strain Range $(\%)$ & $1.8 \mathrm{e} 7$ & $2.4 \mathrm{e} 7$ & $2.8 \mathrm{e} 6$ & $2.7 \mathrm{e} 5$ \\
\hline Expected Cycles to Failure & \multicolumn{5}{|c|}{} \\
\hline
\end{tabular}

Figure 20. Results of ANSYS simulation of tungsten-copper coatings for on/off gyrotron operation 
Results for swept operation were similar to previous results with an order of magnitude greater lifetime for the coatings than for pure copper. These results are presented in Figure 21.

\begin{tabular}{|l|c|c|c|c|}
\hline Case & 1 & 2 & 3 & 4 \\
\hline Coating Thickness (in) & 0 & 0.020 & 0.050 & 0.100 \\
\hline Coating Material & $\mathrm{W}$ & $\mathrm{W}$ & $\mathrm{W}$ & $\mathrm{W}$ \\
\hline Power Density $\left(\mathrm{kW} / \mathrm{cm}^{2}\right)$ & \multicolumn{5}{|c|}{2.55} \\
\hline Peak Temperature $\left({ }^{\circ} \mathrm{C}\right)$ & 304.9 & 371.9 & 451.7 & 538.9 \\
\hline Copper Strain Range $(\%)$ & 0.0542 & 0.0136 & 0.0140 & 0.0024 \\
\hline Tungsten Peak Stress (MPa) & - & 290 & 221 & 290 \\
\hline Tungsten Yield Stress (MPa) & \multicolumn{5}{|c|}{$>500$} \\
\hline Expected Cycles to Failure & $>1 \mathrm{e} 7$ & $>1 \mathrm{e} 8$ & $>1 \mathrm{e} 8$ & $>1 \mathrm{e} 8$ \\
\hline
\end{tabular}

Figure 21. ANSYS results for tungsten copper coatings for swept operation

To verify that these coatings could be achieved, Plasma Coating Corp. tested their system with two different ratios of tungsten and copper. The concern was that the large difference in melting temperatures would prevent proper deposition. Fortunately, the coatings were performed without any problems. Figure 22 shows four coated copper plates with different coating constituents and thicknesses.

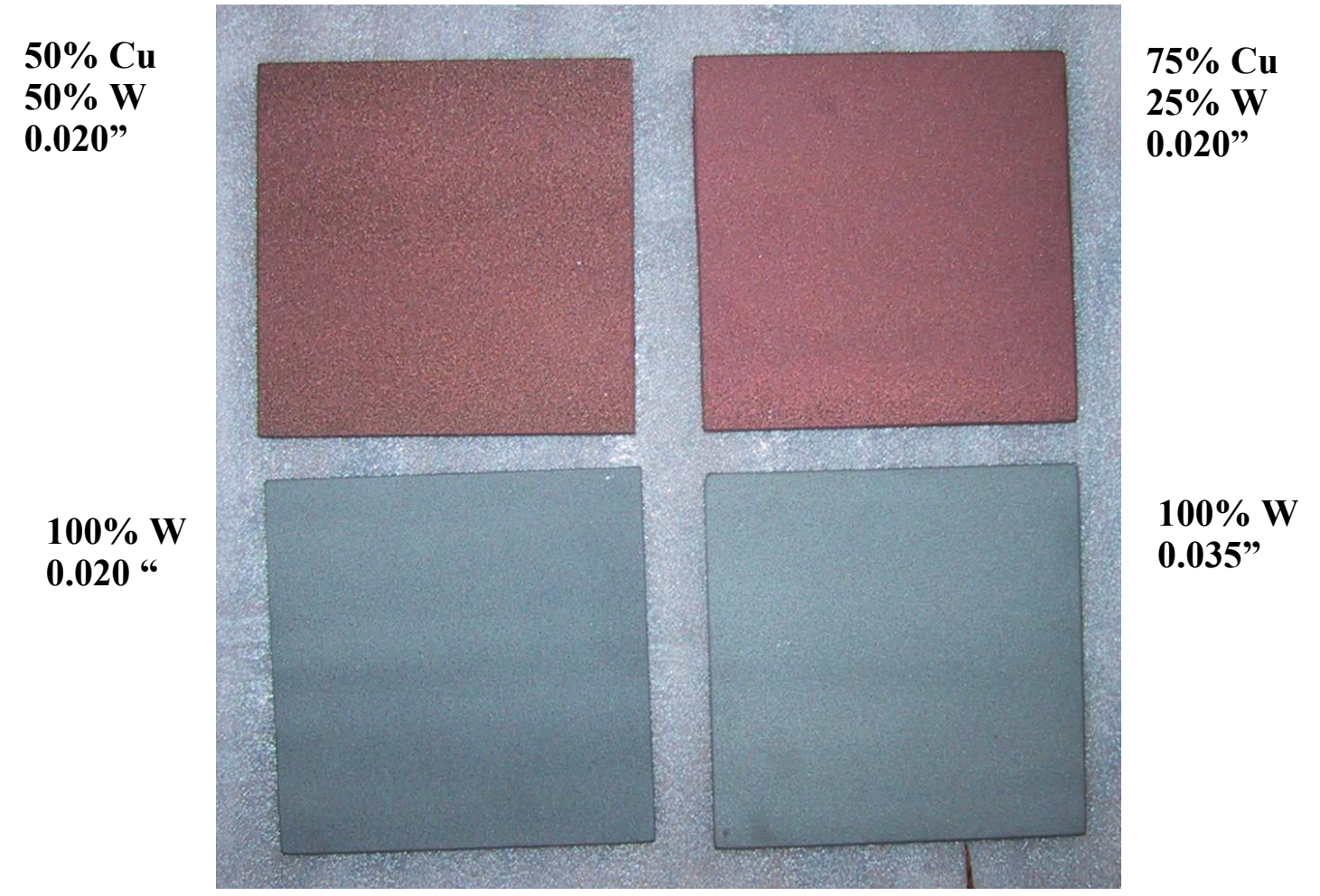

Figure 22. Plasma coated copper plates. The coating ratios and thicknesses are indicated. 
A number of questions remain, however, before plasma coatings could be implemented into gyrotrons. The cyclic performance has not been experimentally verified. It's unclear how accurately the thermal models simulate the actual bonds created during the plasma coating process. It will be necessary to verify that these surfaces can withstand cyclic thermal deposition similar to that in collectors. This experiment is planned for the Phase II program.

\subsection{Develop a real-time diagnostic for collector monitoring during operation}

The Phase I proposal described a technique for monitoring real time collector temperature profiles using microwave reflectometry. The potential advantage of this technique is that it can be used while the gyrotron is operating and interlocked to the power supplies to prevent collector failures due to excessive temperatures. A test device consisting of a copper center conductor inside an alumina cylinder was constructed. This cylinder was assembled inside a brass outer conductor with N-type connectors on each end. Figure 23 shows a photograph of the device.
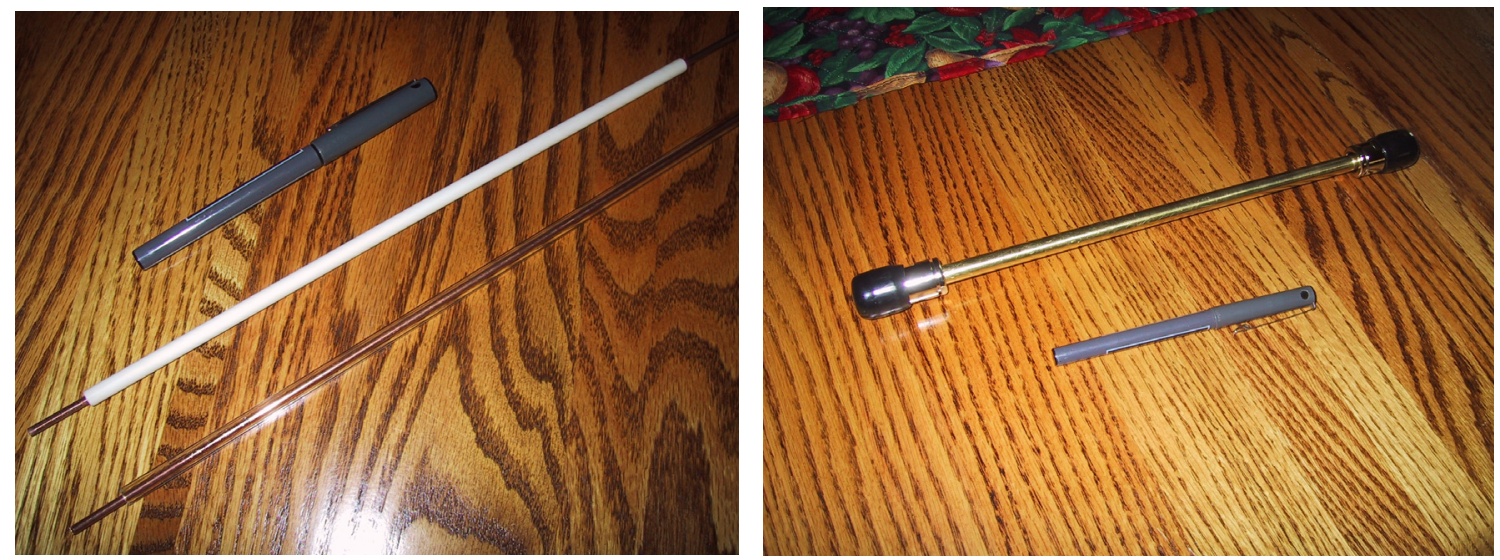

Figure 23. Photograph of copper rod through the alumina cylinder (white) and the competed test fixture with $\mathrm{N}$-type connectors

To test the concept, resistance thermal devices (RTDs) were attached along the rod to measure the temperature profile. Soldering irons connected to VariACs provided heat sources to generate temperature peaks along the length of the rod. Figure 24 shows a photograph of the setup. Network analyzer data was recorded (S11, S12) from $100 \mathrm{MHz}$ to $3 \mathrm{GHz}$ for several experimental temperature profiles.

Attempts to extract temperature from the Network Analyzer data failed. It appeared to be a problem with the mathematical model used to analyze the measurements. While the model worked well on simulated data, it failed to converge for the measured data. To test the model, parameters in the simulated data were very slightly perturbed. The mathematical model became unstable and produced nonsensical results. While the technique, in theory, should work, modifications to the model will be necessary to eliminate this inherent, numerical instability. CCR is continuing to investigate this technique using its own funds. If the problem(s) can be resolved, the technique will be revisited in the Phase II program. 


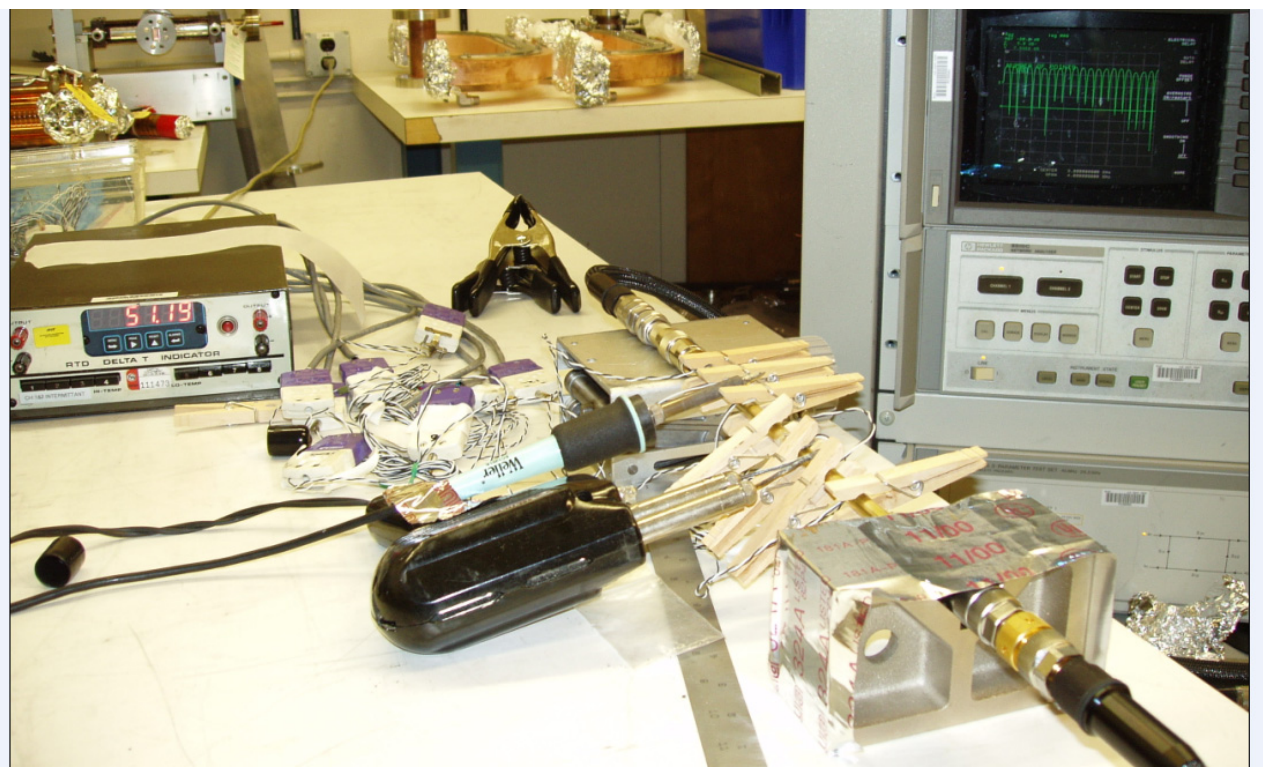

Figure 24. Test setup for reflectometry temperature profile measurement experiment

\subsection{Design a facility for testing collectors in the Phase II program.}

CCR personnel worked with CPI engineers to generate the preliminary design of a facility for high power testing of collector surfaces. No capability currently exists for exposing test surfaces to high power density, electron beams. Consequently, the Phase I program explored a system generating a continuous or pulsed electron beam of sufficient size and energy density. Figure 25 shows a solid model of the proposed device, consisting of an electron gun, curved drift tube, and test chamber. The drift tube is curved to avoid ions or debris blasted from the test surfaces from reaching the cathode. A bending magnet will be inserted around the curved drift tube. This magnet was designed with assistance from Stanford Linear Accelerator Center personnel. Philipp Borchard assisted with design of the test chamber to ensure material surfaces relevant to practical collectors could be tested.

The end plate for the test chamber is removable for inserting test structures. Figure 26 shows a view of the end plate. Test structures are mounted on the copper cooling plate and captured by a stainless steel flange. The cooling plate was designed for flow rates similar to those in gyrotron collectors. The system allows users to quickly remove and insert materials for testing.

The electron gun is from a $17 \mathrm{~kW}$ CW, L-Band klystron designed and built by CCR. This gun is air cooled, which will facilitate operation in CCR's laboratory. The gun can operate continuously or pulsed. Pulsed operation will be crucial for cyclic testing of materials.

The test device would be ideal for testing the plates fabricated in Task 2.4. This would allow performance comparison between various combinations of coatings and base materials. It would also ensure that coated surfaces do not suffer deterioration, such as delamination or separation, following extensive cyclic operation. 


\subsection{Summary of the Phase I Program Results}

The program was tasked with determining feasibility of developing gyrotron collectors capable of reliable, long lifetime operation on $1.5 \mathrm{MW} \mathrm{CW}$ gyrotrons. Two approaches were explored. Task 2.2 investigated alternative designs to reduce the peak power densities and eliminate axial beam sweeping. These results confirmed that designs could be achieved with power densities compatible with continuous operation without beam sweeping. Therefore, the feasibility of this approach was clearly demonstrated.

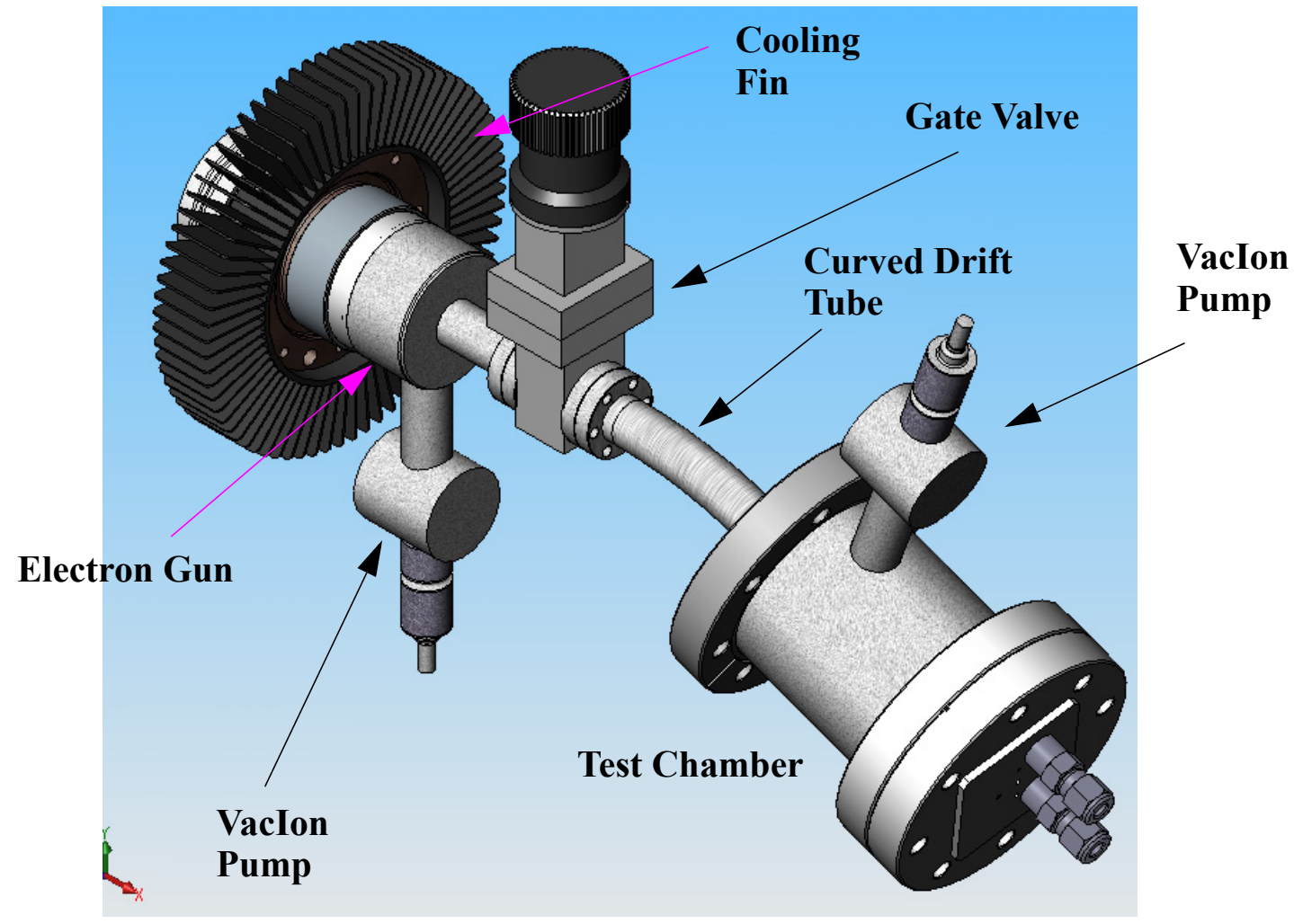

Figure 25. Preliminary design of test facility for high power testing of collector surfaces. The bending magnet is not shown.

The second approach involved application of plasma coatings to copper collectors to provide increased strength for cyclic operation present in axial beam swept collectors. The thermal analyses indicated that cyclic lifetimes using pure tungsten coatings could be increased by approximately one order of magnitude over pure copper, though with decrease of lifetime for on/off operation. A plasma coating process was developed for plasma spraying of a tungsten - copper mixture to address the on/off operating performance. These results indicated that the proper coating with an equal quantity of tungsten and copper could increase both the on/off and swept lifetimes. While feasibility was demonstrated, a number of questions related to the long term cyclic stability of plasma coated surfaces remain. Experimental testing will be required before this technology can be implemented into a collector. The test device designed in Task 2.6 would provide this testing capability. The Phase I results indicate that this approach is feasible for increasing cyclic lifetime in axially swept collectors. Analysis indicates an order of magnitude increase in lifetime. 
The Phase I program also investigated feasibility of a diagnostic technique for monitoring collector temperatures during full power operation. Results were described in Section 2.5 and indicate that feasibility has not yet been demonstrated. Testing will continue until definitive results are obtained.

\section{References}

[1] "Status of DOE/GA Gyrotron Activities at CPI," Gyrotron Community Meeting, Palo Alto, CA, July 13, 2005

[2] R.W. Callis, C. Baxi, Gorelov, "Collector thermal evaluation by General Atomics," Gyrotron Community Meeting, Palo Alto, CA, July 13, 2005.

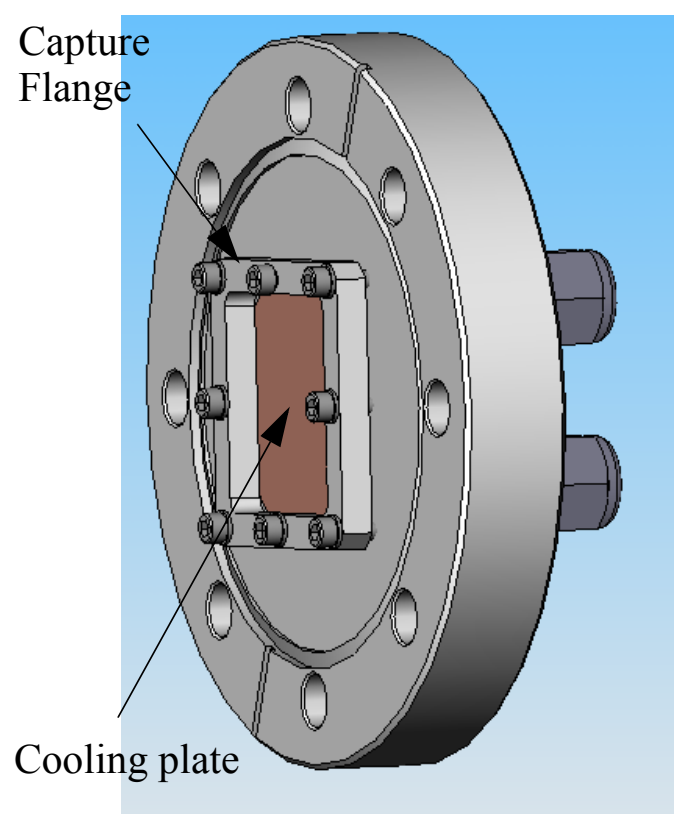

Figure 26. Test device flange

[3] "Development of a Multi-Stage Depressed Collector System for Gaussian Mode Gyrotrons." U.S. Department of Energy Small Business Innovation Research Grant Number DE-FG0397ER82342, September 1997 through December 2002.

[4] Advanced Backward Wave Oscillators, NASA contract No. NAS3-01014, Administered by NASA Glenn Research Center, January 2001- January 2004,

[5] R. Lawrence Ives, "Depressed Collector for Electron Beams, U.S. Patent \# 2003/ 0122491 A1, July 3, 2003.

[6] Manfred Thumm, private communications, IRMM 2006 Conference, private communications, September 2006.

[7] Kevin Felch and Monica Blank, private communication, March 2007. 